\title{
El aprendizaje reflexivo en la formación inicial de maestros/as: de la experiencia a la integración y síntesis de los contenidos
}

\author{
Reflexive learning in pre-service training of primary \\ school teachers: from experience to assimilation of \\ contents \\ $\mathrm{M}^{\mathrm{a}}$ Teresa COLÉN RIAU, Beatriz JARAUTA BORRASCA y \\ Leyla Claudina CASTRO GONZÁLEZ \\ Universidad de Barcelona
}

Recibido: Junio 2014

Evaluado: Noviembre 2014

Aceptado: Enero 2015

\begin{abstract}
Resumen
Este artículo presenta una experiencia didáctica implementada en el grado de Maestro/a de Educación Primaria de la Universidad de Barcelona. En concreto, el artículo analiza la implementación de la estrategia de los "Rincones de Trabajo" en una asignatura troncal. A través de un proceso de investigación-acción de una duración de tres años, las docentes implicadas han desarrollado una innovación que ha permitido potenciar el trabajo colaborativo y un aprendizaje reflexivo en los estudiantes. En primer lugar, se presentan los referentes teóricos que inspiraron el proyecto y orientaron buena parte de las acciones realizadas por el equipo docente. Tras ofrecer información detallada del desarrollo práctico de la experiencia, se relata el proceso de recogida de datos y se presentan los resultados. Para la valoración de la experiencia se utilizaron diversas estrategias de recogida de información como el cuestionario, la observación participante y los diarios del profesorado. Los resultados muestran, entre otros aspectos, que los "rincones de trabajo" brindan condiciones didácticas viables y adecuadas para la articulación de conocimientos teóricos y prácticos, para la construcción de conocimientos profesionales y para el inicio del ejercicio de una práctica docente reflexiva.
\end{abstract}

Palabras clave: Enseñanza Superior, formación de docentes de primaria, innovación pedagógica, proceso de enseñanza, método de enseñanza.

\begin{abstract}
This article presents an educational experiment carried out in the Primary School Teaching Degree at the University of Barcelona. Specifically, the article analyses the application of the "Work Corners" approach in a core subject. In a three-year action research process, trainers put into practice an innovation which enabled them to boost cooperative work and reflexive learning among trainees. Firstly, the theoretical model underpinning the project and guiding many of the actions carried out by the training team is presented. After providing detailed information on the practical development of the experiment, the data-gathering process and its
\end{abstract}


results are shown. Various information-gathering strategies were used in assessing the project, such as a questionnaire, participant observation, and teachers' diaries. The results demonstrate, amongst other things, that "work corners" offer viable and appropriate educational conditions for the articulation of theoretical and practical knowledge, for building professional knowledge, and therefore, the beginnings of a reflexive teaching practice.

Keywords: higher education, elementary teacher education, educational innovation, teaching process, teaching method.

La paulatina adaptación de los modelos educativos universitarios a los retos derivados del Espacio Europeo de Educación Superior (EEES), ha exigido cambios sustanciales en la función práctica formativa y en las relaciones que se establecen entre alumnado y profesorado. La implementación del crédito europeo, la necesidad de valorar e impulsar el aprendizaje autónomo del alumnado y la importancia de asegurar, desde las instancias universitarias, el desarrollo de un conjunto indispensable de competencias, han requerido la generación de espacios y propuestas pedagógicas que realmente sitúen al alumnado en el centro de la enseñanza y el aprendizaje.

De acuerdo con el modelo educativo propugnado por el EEES, que valora la competencia de “aprender a aprender”, y considerando que el valor esencial de los estudios de formación del profesorado reside en que los estudiantes "aprendan a enseñar”, mostramos en las siguientes páginas el análisis de una experiencia llevada a cabo en el grado de Educación Primaria de la Universidad de Barcelona. En concreto, este artículo describe un proceso de investigación-acción centrado en el diseño y desarrollo de la estrategia formativa de los "Rincones de trabajo" en una asignatura obligatoria. La experiencia que presentamos ha pretendido contribuir a dos grandes retos: la formación reflexiva de maestros y el aprendizaje cooperativo de los estudiantes.

Exponemos aquí los fundamentos teóricos de la experiencia y una síntesis del proceso seguido por el equipo docente. El artículo finaliza con un conjunto de valoraciones que resitúan el alcance formativo de los "Rincones de trabajo" en el contexto concreto de la titulación de Magisterio, considerando el entramado de expectativas y vivencias protagonizadas por los estudiantes y profesoras participantes.

\section{Fundamentación teórica}

Uno de los motivos que nos llevó a proponer los rincones como estrategia de enseñanza fue el pretender superar la dicotomía teoría-práctica, todavía predominante en la formación inicial de maestros (Pérez, 2010; Zeichner, 2010; Álvarez, 2013). Desde los planteamientos de Schön $(1983,1992)$ sobre la importancia del pensamiento práctico, sabemos que los profesionales desarrollan un conocimiento personal como consecuencia de su actividad, que les ayuda a interpretar los episodios prácticos de su profesión. El conocimiento práctico no es una mera reproducción de los esquemas y rutinas empíricas que modelan la práctica profesional, sino que supone una integración de la teoría y la práctica mediante actividades de análisis y reflexión. La formación de profesores tendría que atender a esta caracterización epistemológica que concibe al 
maestro como un profesional práctico (Flores, 1998) y configurar e implementar currículos que contemplaran y apreciaran la creación de contextos de pensamiento y acción. En esta línea de argumentación, Pérez Gómez (2010) se refiere especialmente a un currículum que se construya sobre los intereses, fortalezas y pensamiento práctico previo de los estudiantes y que transcurra a través de actividades auténticas que provoquen la implicación y experiencia educativa del estudiante.

Hemos tenido también muy presente que la enseñanza reflexiva requiere hacer al alumnado partícipe de la misma. Ésta no puede entenderse como un proceso unidireccional; adquiere pleno sentido cuando se entiende en relación al aprendizaje y cuando se otorga al alumnado un rol activo y comprometido con su formación. Se trata entonces de ayudar al estudiante a resolver situaciones ambiguas y poco claras, tal cual se presentan en el ejercicio profesional. Esto significa ofrecer al alumno oportunidades de realizar actividades, de manera que pueda hacer "cosas" y dialogar sobre lo que surja en el curso de sus acciones (Dewey, 1916). Estos principios han orientado el diseño y el desarrollo de los rincones de trabajo.

La estrategia de los rincones nos ha permitido, además, trabajar otras competencias con el alumnado: trabajo en equipo, habilidades de coordinación y gestión de grupos, organización y distribución de tareas, liderazgo, empatía, escucha activa y evaluación y regulación del progreso de aprendizaje. Todas ellas, competencias que son cruciales para el ejercicio docente y desarrollo profesional del profesorado. El trabajo en equipo, como señala Antúnez (1999) refiriéndose a la profesión docente, se presenta como un mecanismo que puede proporcionar una atmósfera que anime a los alumnos a trabajar con entusiasmo y aumentar el autoconcepto y la autoestima del profesorado y su sentimiento de pertenencia respecto al centro escolar.

El trabajo en equipo y otras actividades cooperativas de aprendizaje deben tener un lugar privilegiado en la enseñanza universitaria porque, en comparación con otros métodos de enseñanza, el aprendizaje cooperativo es para los estudiantes una de las maneras más eficaces de maximizar su propio aprendizaje y los logros académicos de sus compañeros (Saleh, 2011, Barba et al. 2012; Tran y Lewis, 2012) Las formas de trabajar en equipo son muchas y variadas y se crean con finalidades muy diversas (Barkley, Cross y Howell, 2007). A la hora de conformar los equipos hemos tenido en cuenta los criterios que, según Johnson, Johnson y Holubec (1999), caracterizan los grupos de trabajo eficaces:

- Interdependencia positiva: El éxito individual de las personas está vinculado al éxito del grupo. Todos los integrantes están obligados a confiar en los otros para conseguir el objetivo.

- Interacción promotora: Los estudiantes se ayudan y apoyan entre sí. Comparten recursos e estimulan la implicación y esfuerzo de los demás.

- Responsabilidad individual y de grupo. El grupo es responsable de lograr sus objetivos a la vez que cada miembro se compromete a realizar su parte del trabajo. 
- Desarrollo de las competencias de trabajo en equipo: Los estudiantes aprenden cuestiones académicas a la vez que competencias de trabajo en equipo.

- Interacción constante entre los estudiantes: Los estudiantes interactúan entre ellos con elevada frecuencia para discutir lo que están trabajando, identificar los contenidos que deben aprender y los procedimientos que deben aplicar, revisar el trabajo realizado, ofrecer apoyo, asistencia, estímulo, etc.

- Valoración del grupo: Los estudiantes evalúan el funcionamiento del grupo y su productividad.

En base a las aportaciones anteriores, la experiencia de los rincones parte de la idea de que los estudiantes fundamentalmente aprenden a través de un proceso activo, cooperativo y autorregulado, dirigido a hallar significados y a construir conocimientos que surgen de sus experiencias en situaciones reales (Piqué, Comas y Lorenzo, 2010).

\section{Descripción de la modalidad: los rincones de trabajo}

Los rincones de trabajo se inscriben en el marco de una renovación pedagógica de principios del siglo XX, nacida de la crítica a la enseñanza tradicional de la época. El movimiento de la "Escuela Nueva" reconocía al alumnado y su acción como núcleos de toda actividad de enseñanza y aprendizaje, siendo los problemas de la vida cotidiana el marco donde se inscribía y se desarrollaba la acción. Los rincones de trabajo se plantean como una metodología que impulsa una forma de enseñanza estimulante, flexible y dinámica que permite ser adaptada a diferentes contextos. El trabajo por rincones en el aula es una forma de organización que permite en el alumnado el desarrollo de hábitos elementales de trabajo, el establecimiento y cumplimiento de normas y, en especial, el desarrollo de su autonomía. Esta metodología se basa en los modelos o paradigmas ecológicos de autores como Doyle o Bronfenbernner. El aula se divide en diversos espacios de trabajo preorganizados por el maestro y que, previa elaboración de normas de uso, los propios niños administran. Son espacios delimitados y concretos, ubicados en las aulas o su entorno más próximo donde los alumnos trabajan de manera simultánea y alrededor de un único proyecto o unidad de programación (Rodríguez Torres, 2011).

La metodología por rincones consiste en proponer actividades variadas que es necesario finalizar en un tiempo concreto. Durante este tiempo, cada niño puede escoger el rincón por el que desea comenzar, sabiendo que durante el período de tiempo que estipule el maestro tendrá que participar en todos los rincones. Los rincones requieren de un conjunto de condiciones para su buen funcionamiento (Herrera, 2009):

- Haber finalizado y corregido la tarea curricular que se esté trabajando.

- La elección del rincón es libre.

- Acudir al rincón deseado si éste tiene sitio disponible.

- Anotar en el registro personal en qué rincón se ha trabajado y qué se ha hecho.

- Recoger al finalizar la actividad los materiales utilizados. 
- Trabajar con autonomía.

- Moverse en silencio.

- Aceptar las normas de agrupamiento para algunas de las actividades propuestas.

- Mantener los rincones en orden.

- No preguntar al maestro/a a no ser que sea estrictamente necesario.

- Si un trabajo no se ha terminado, se guarda y se puede continuar en otro momento.

Los aprendizajes que, en Educación Infantil y Primaria, pueden obtenerse a través de los rincones de trabajo son significativos y pueden abarcar diversos ámbitos del desarrollo global del niño (ámbito cognitivo, psicomotor y afectivo-social). En la escuela, pueden organizarse una variedad ilimitada de rincones. Todo depende de la creatividad del maestro, de los recursos disponibles y de las características de los alumnos. El rincón de las construcciones, de la cocina, de la naturaleza viva, del mercado, del día y la noche, del circo, de los cuentos... son algunos ejemplos de rincones.

El trabajo por rincones en estas etapas se diferencia de manera sustancial del trabajo por rincones en la enseñanza universitaria y en el ámbito de la formación inicial de maestros. En el primer caso, el principio de aprendizaje que sustenta el rincón es el de "descubrimiento" y se produce cuando el maestro proporciona al alumno un conjunto de recursos para que él, por sí mismo, descubra lo que desea aprender. En el caso de la enseñanza universitaria, predomina el principio de "globalización" que se da cuando el estudiante lleva a cabo un aprendizaje consciente y relacionado en el que encuentra nuevo conocimiento y en el que establece conexiones entre éste y los conocimientos e informaciones de los que dispone. El principio de globalización no se produce por la simple acumulación o adición de nuevos elementos a la estructura cognitiva del estudiante sino por la integración que establece entre los conocimientos que posee y los nuevos. Otra intención pedagógica es que las actividades propuestas permitan establecer una conexión e interacción entre el conocimiento académico, las ideas previas y creencias del alumnado y la realidad de la educación escolar juntamente con la variabilidad de contextos en los que ésta se produce.

La experiencia de los "Rincones de Trabajo" se ha realizado durante dos cursos académicos en la asignatura de "Planificación, diseño y evaluación del aprendizaje y la actividad docente" del grado de Educación Primaria. Se trata de una asignatura troncal de seis créditos que se imparte en el segundo semestre del primer curso del grado y que está asignada al área de conocimiento de Didáctica y Organización Escolar. Se imparte semanalmente, tres horas en dos sesiones. El objeto de la asignatura es ayudar a los estudiantes a comprender, planificar y dinamizar la vida en el aula y del grupo clase, en su complejidad social, relacional y educativa. 


\section{Metodología de investigación}

El enfoque adoptado en el proceso investigador responde a una metodología cualitativa, concretamente a una investigación-acción. La investigación-acción supone entender la enseñanza como un proceso de investigación que integra el análisis y reflexión del profesorado en torno a las experiencias que realiza en su práctica docente. Se trata de un tipo de investigación protagonizada por los profesores que surge de los interrogantes que se plantean a partir de su experiencia cotidiana (Cochran-Smith y Lytle, 2003). Es una metodología que permite analizar y recuperar los saberes y creencias del profesorado a la vez que generar conocimiento pedagógico a través de procesos de observación y reflexión sobre la propia práctica. El objetivo último de esta metodología es la mejora de la práctica educativa.

El proceso de investigación-acción se concibe como una espiral de ciclos que se retroalimentan entre sí y se componen de cuatro fases: planificación, acción, observación y reflexión. Su carácter cíclico implica un "vaivén” (espiral dialéctica) entre la acción (praxis) y la reflexión (teoría), de manera que ambos momentos quedan integrados y se complementan (Latorre, Arnal y Del Rincón, 1996). La siguiente figura recoge información relevante acerca de las fases seguidas en el proyecto:

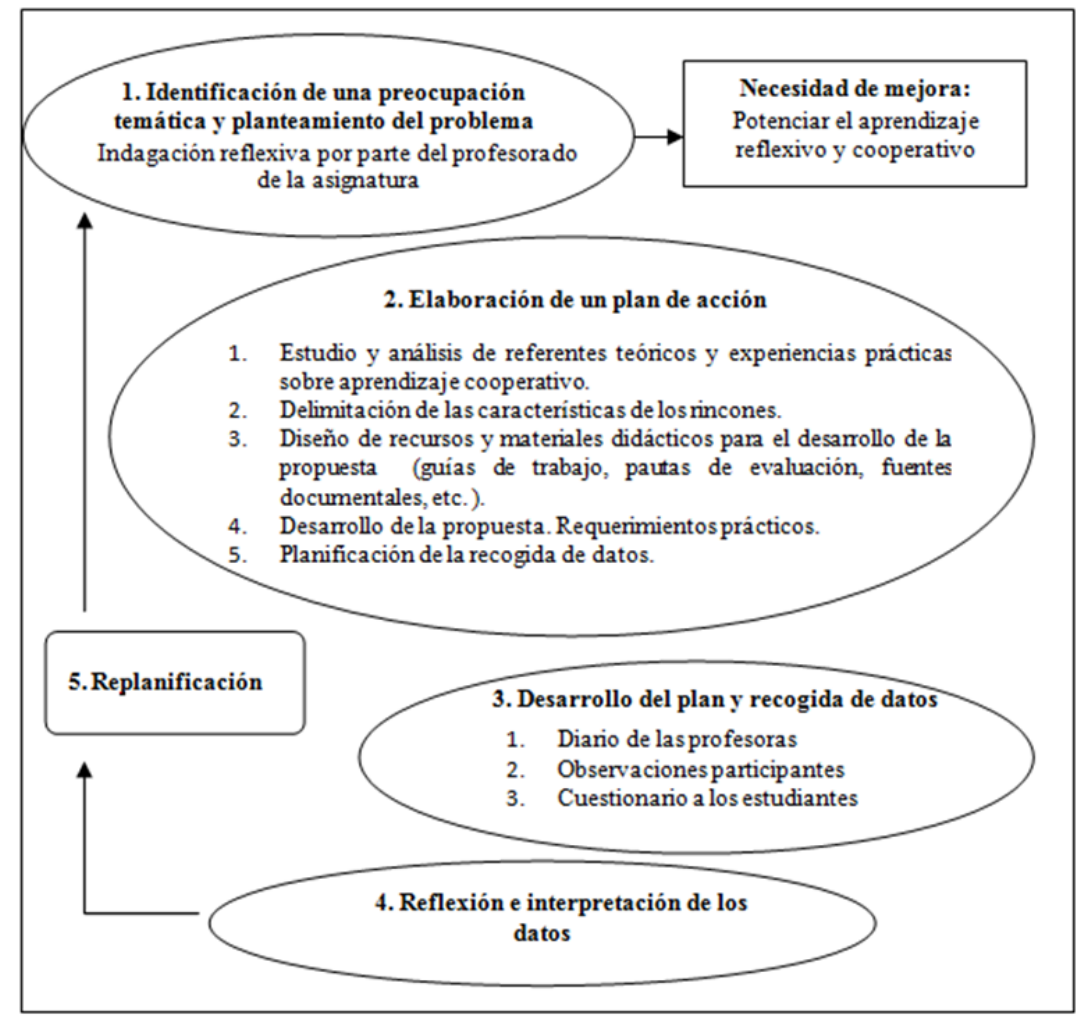

Figura 1: Desarrollo de la investigación-acción (Adaptación de Sandín, 2003). 
En nuestro caso, el compromiso que como profesoras teníamos con la comprensión y la mejora de nuestra práctica docente fue el origen del proyecto. Durante el curso 2009-2010 iniciamos un proceso de reflexión, debate y consenso, cuyo resultado supuso el inicio de la experiencia. Fue en el curso 2010-2011 cuando empezamos el proceso de investigación-acción cuya finalidad última fue introducir en el aula modalidades de enseñanza que reforzaran el aprendizaje reflexivo y cooperativo de los estudiantes, en concreto los rincones de trabajo.

El trabajo de campo se extendió durante los cursos 2010-2011 y 2011-2012 y las principales estrategias de recogida de información fueron la observación participante, los diarios del profesorado y los cuestionarios abiertos al alumnado. En el proyecto participaron dos grupos de estudiantes por curso, siendo finalmente un total de cuatro grupos y 258 estudiantes. Participaron también tres profesoras de la asignatura de “Planificación, diseño y evaluación del aprendizaje y la actividad docente”.

La modalidad de observación adoptada en la recogida de datos fue la participante. Las profesoras a medida que interveníamos en el aula recogíamos información acerca del desarrollo de la experiencia. Para ello utilizamos diarios de campo, en los que tratamos de recoger las observaciones y reflexiones que hacíamos acerca de los problemas que surgían en clase y de nuestras reacciones frente a ellos (Elliott, 1990) Los diarios contenían observaciones, reflexiones, interpretaciones, hipótesis y explicaciones.

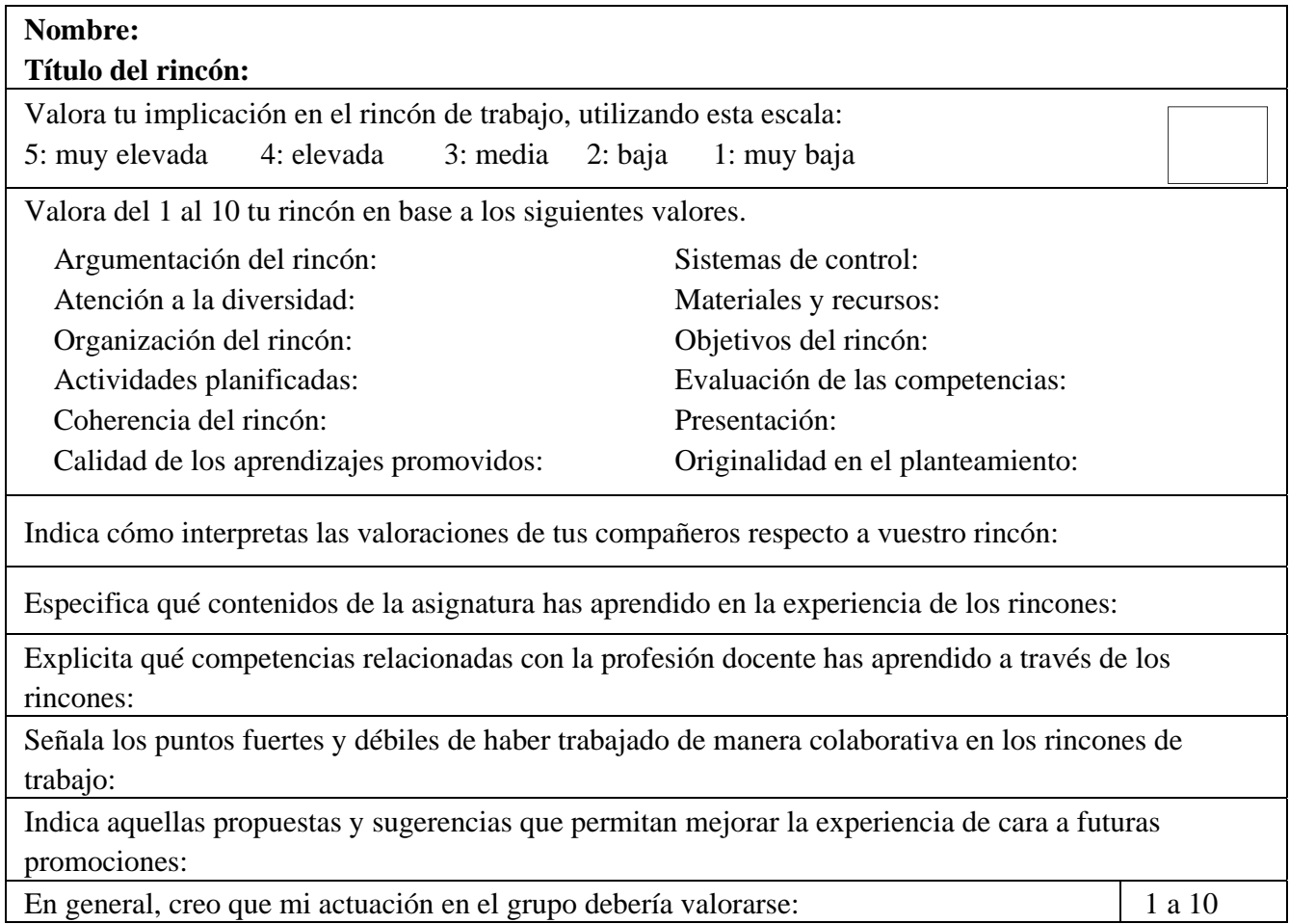

Tabla 1. Cuestionario de autoevaluación de los estudiantes. 
En último lugar, el cuestionario utilizado para la autoevaluación individual de los estudiantes se convirtió en un material importante de obtención de información, que nos ha permitido valorar la experiencia en su conjunto y conocer aquellos aspectos que requieren ser mejorados. El cuestionario (Tabla 1) contenía preguntas cerradas y preguntas abiertas y se dirigía fundamentalmente a conocer el funcionamiento de los equipos de trabajo y aquellos aprendizajes que los estudiantes habían logrado por su participación en los mismos:

\section{Resultados}

A continuación presentamos los resultados que aluden a la satisfacción de los estudiantes y a los aprendizajes y competencias que han alcanzado una vez finalizada la experiencia. De igual manera, recogemos algunas conclusiones y propuestas de mejora de cara a futuras implementaciones.

Para una mejor comprensión de los resultados obtenidos, el sistema de notación utilizado para citar las unidades de significado que sustentan las interpretaciones realizadas por el equipo investigador incluye una referencia numérica mediante la cual se identifica al alumno/a o al profesor/a (AM1: Alumno de Magisterio 1, P1: Profesor 1) Al final, especificamos la fuente de información, siendo "A" información extraída de la autoevaluación, "O" de observaciones y "DC" equivalente a fragmentos de los diarios de campo del profesorado.

\section{Valoración de la experiencia}

Una vez finalizada la asignatura, los alumnos manifestaron estar altamente satisfechos con la actividad de los rincones. Valoraron especialmente su carácter práctico y el hecho de tratarse de una actividad grupal. El buen clima generado en el aula y la posibilidad de transferencia de los aprendizajes obtenidos, aparecieron también como elementos a los que los estudiantes otorgaron un gran valor:

AM12A: "El trabajo por rincones ha sido especialmente importante porque es una de las estrategias que utilizaremos, en un futuro, cuando practiquemos la docencia. Hemos podido diseñar nuestro propio rincón y hemos podido observar los rincones de los compañeros. Ha sido un trabajo muy práctico que hemos realizado en grupo. Ha habido buen clima y hemos podido experimentar muchos de los contenidos trabajados hasta el momento en la asignatura"

El Rincón del Zoo, el de los Reporteros o el del Herbolario fueron algunos de los rincones desarrollados por los estudiantes. El trabajo por rincones, según los alumnos, les había ayudado a adquirir un conjunto de competencias como la autorregulación y autonomía en los aprendizajes. Consideraron que habían asumido un elevado grado de responsabilidad en su proceso de aprendizaje al tener que concretar el tema, las actividades, los tiempos, recursos y evaluación del rincón. La ausencia de límites por parte del equipo docente en algunos aspectos concretos, había contribuido a que los 
estudiantes se sintieran con la libertad suficiente como para explorar en temáticas insospechadas y diseñar actividades y recursos con grandes dosis de creatividad:

A29A: "El no estar predeterminado a hacer algo cerrado y sugerido por la profesora nos ha ayudado a hacer volar la imaginación. Esto nos ha motivado a buscar información de experiencias de rincones ya llevadas a cabo en escuelas, arriesgarnos con el tema, considerar si era pertinente para el ciclo y nivel escogido y elaborar los materiales"

A la hora de valorar en su justa medida esta "ausencia de límites", es oportuno señalar que, lejos de un aprendizaje llevado a cabo sin ningún tipo de indicación por parte del equipo docente, nuestra intervención en el aula se centró en generar las condiciones necesarias para que los estudiantes fueran los encargados de dirigir su propio proceso de aprendizaje. Para ello, conceptualizamos en el aula el trabajo por rincones como estrategia de aprendizaje y elaboramos un conjunto de materiales dirigidos a apoyar los pasos que los estudiantes debían dar para diseñar su rincón. Las acciones llevadas a cabo por el equipo docente fueron las siguientes:

- Elaborar orientaciones teóricas de forma oral, escrita y audiovisual acerca de qué es un rincón de trabajo para ciclo inicial, para qué sirve, cómo se utiliza, ejemplos de rincones reales, ventajas e inconvenientes, condiciones para su elaboración, etc.

- Elaborar una pauta con orientaciones para la planificación, elaboración, evaluación del rincón, coevaluación y autoevaluación del proceso.

- Coordinar, guiar y motivar a los alumnos antes y durante el ejercicio.

- Realizar tutorías, grupales e individuales, que garantizaran un proceso rico y reflexivo.

- Coordinar la participación en las presentaciones.

- Preparar el proceso de elaboración cooperativo de rúbricas para la evaluación del trabajo.

- Dirigir el proceso de coevaluación conjunta, garantizando aportaciones constructivas y la interiorización por parte del grupo de los criterios de evaluación.

- Valoración de los aprendizajes y tareas llevadas a cabo por los estudiantes.

De manera complementaria y a medida que la experiencia iba avanzando, intentamos tender puentes entre los contenidos de la asignatura y el diseño de los rincones; entre lo que los estudiantes conocían por haber participado en el primer curso de grado y los nuevos conocimientos derivados de la actividad. En este sentido, el trabajo por rincones se nos presentó como una opción inmejorable para integrar y sistematizar contenidos de la asignatura que se habían trabajado de manera proposicional y desligados de las situaciones prácticas del magisterio. Competencias específicas como la planificación de la enseñanza, el diseño de actividades didácticas de carácter lúdico, el tratamiento de la diversidad y la evaluación de los aprendizajes 
fueron abordadas de manera extensa a través de los rincones de trabajo. Estas cuestiones fueron recogidas por una de las profesoras en su diario de campo:

P3DC: "La dificultad está en orientar el trabajo bajo los parámetros de una asignatura compleja en la que se trabajan contenidos que tienen que ver con la planificación, metodología y evaluación docentes y conseguir que los estudiantes vuelquen todo aquello que han aprendido en su propuesta de rincón. Las tutorías y las sesiones de evaluación en el aula se han convertido en espacios interesantes en los que ayudar a los estudiantes a visualizar todos los aprendizajes que han logrado en la signatura y ahora ponen en juego para diseñar el rincón”

Cabe señalar que estos aprendizajes, relativos a ciertas competencias específicas de la asignatura, también fueron reconocidos por parte de los estudiantes:

AM9A: "Estamos contentos de haber podido trabajar en un rincón que nos ha aportado una ampliación de conocimientos sobre metodología, una aplicación práctica sobre la misma, una planificación de un conjunto de actividades, un análisis de la tarea para saber con qué estamos trabajando exactamente y por otro lado, unos momentos distendidos donde poder aprender de forma indirecta y significativa"

Además de la competencia de aprender a aprender y ciertas competencias específicas, los estudiantes manifestaron haber adquirido otras habilidades. De manera reiterada, citaron las habilidades de análisis y síntesis, de comunicación y de trabajo en equipo. La siguiente matriz recoge información al respecto:

\section{Competencias}

Competencia de aprender a aprender. Fomento de la autonomía en el aprendizaje y construcción de nuevos conocimientos al otorgar a los estudiantes la responsabilidad de gestionar y organizar el trabajo por rincones, tomar decisiones en torno a los criterios de búsqueda de fuentes de información y preparar el material necesario para las sesiones de discusión y reflexiones realizadas en el aula. Capacidad para organizar el tiempo y distribuir de manera adecuada el conjunto de tareas individuales y grupales que la actividad exija.

Competencias de análisis y síntesis. Relacionadas con la capacidad de identificar los elementos más relevantes de la asignatura y transferirlos al diseño de un rincón, con la capacidad de organizar la información utilizada en el diseño y transmitirla, de forma sintética, a los compañeros.

\section{Información procedente de los cuestionarios}

AM4A: "He aprendido a regular mi tiempo, a distribuirlo entre aquellas tareas que debía hacer de manera individual y el tiempo que debíamos dedicar al trabajo grupal, para lograr hacer las dos de la mejor forma posible"
AM51A: "Me he dado cuenta de que la mejor manera de trabajar es dedicando la mayoría del tiempo a recoger información básica que utilizarás y a la propia organización de trabajo, y que una vez tienes esto la redacción del trabajo es más rápida y se trata de extraer las ideas principales" 


\begin{abstract}
Competencias comunicativas y escritas. Capacidad de escribir con un lenguaje adecuado y con corrección lingüística en el trabajo escrito vinculado al rincón y capacidad para presentar y defender, con claridad, las premisas asociadas al rincón.
\end{abstract}

AM44A: "El hecho de tener que presentar el trabajo ante los compañeros me ha ayudado a perder el miedo y a intentar buscar las mejores palabras para explicar en 15 minutos nuestro rincón"
Desarrollo de competencias de trabajo en equipo. Relacionadas con habilidades de gestión de grupo, organización y distribución de tareas, liderazgo, acompañamiento y estímulo del trabajo realizado por los demás y evaluación y regulación del progreso de aprendizaje.
AM21A: "Sobretodo he aprendido a trabajar en equipo. En el grupo, sólo conocía a dos personas de las cinco que éramos. Esto me puso a prueba. El hecho de trabajar con desconocidos hace que esta capacidad de integrarse, comunicarse, etc. se desarrolle en gran medida"

Tabla 2. Competencias logradas en la experiencia.

Finalmente, y pese a los buenos resultados obtenidos, algunos aspectos requieren ser revisados. Así por ejemplo, algunos estudiantes solicitaron una mayor coordinación entre los grupos en cuanto a las temáticas abordadas en los rincones, una mejor distribución del tiempo en las presentaciones (específicamente del tiempo del que disponen los alumnos para participar en las actividades de cada rincón) y una organización del espacio físico del aula más adecuada para la realización de las presentaciones:

AM4A: "Ha sido muy interesante pasar por todos los rincones y poder disfrutar todos, pero puede que hubiera sido mejor si hubiera habido más tiempo"

AM3A: "Sería conveniente conocer los rincones de los compañeros de la clase con anterioridad para poder coordinarse y no repetir temáticas"

\section{Trabajo por rincones y procesos de reflexión sobre la práctica}

Uno de los aspectos más reiterados por los estudiantes fue el relativo a la adquisición de habilidades para el desarrollo de un conocimiento vinculado a la profesión, en concreto habilidades de reflexión e interpretación de la propia práctica docente y de los ambientes de aprendizaje. Habilidades que, como reconocieron los estudiantes, se activaron en el momento en el que tuvieron que otorgar un significado a aquellos contenidos teóricos y "en abstracto" que poseían para dar forma y recrear una situación simulada en la que debían actuar como maestros/as:

AM8A: "El hecho que utilicemos la práctica como herramienta principal de aprendizaje ha hecho que desarrollemos más esta competencia y seamos capaces de reflexionar mucho más todo aquello que estamos haciendo en clase. Hemos planificado, hemos diseñado y hemos evaluado actividades que es lo que en un futuro haremos en las escuelas. Tenemos unos saberes y los hemos podido ejercitar, manipular para simular una experiencia de rincones" 
Como consideran autores como Griffin (2003), Perrenoud (2001) o Russell (2005), la adquisición de competencias reflexivas es uno de los objetivos de la formación inicial de maestros. Junto a ello, se hace necesario aproximar a los estudiantes a situaciones propias de la práctica profesional, ayudándoles a otorgar un significado renovado a sus conocimientos a través de la práctica. En nuestro caso, ésta era una pretensión ambiciosa. Trabajábamos con alumnos de primer curso y su background disciplinar y experiencial como estudiantes de magisterio no era elevado. Poseían además un saber asociado a la práctica profesional frágil, pleno de intuiciones y todavía sin confrontar en situaciones reales de enseñanza. Siguiendo a Korthagen (2010), quien considera contraproducente presentar a los futuros maestros un modelo de reflexión al inicio de la formación sin suficiente experiencia docente, decidimos plantear la reflexión simplemente como contemplación de la práctica, también como un ejercicio de autoconocimiento. La reflexión entonces se volvía beneficiosa si estimulaba a los estudiantes a reflexionar sobre las propias experiencias vividas en clase en base a sus preocupaciones personales (Korthagen, 2010) Bien planteada, podía también conducir a los alumnos a interpretar los saberes pedagógicos -en ocasiones vulgares y poco reconocidos- que utilizaban en una situación de enseñanza particular.

Para impulsar estos procesos de análisis e interpretación, recurrimos al modelo de aprendizaje reflexivo experiencial de Kolb (1984), que parte de una situación real y a través de un proceso en el que los componentes principales son la reflexión sobre la experiencia y la retroacción, se llega a la mejora de la actividad docente (Imbernon y Medina, 2008) Este modelo, adaptado al trabajo por rincones, se concretó en las siguientes etapas:

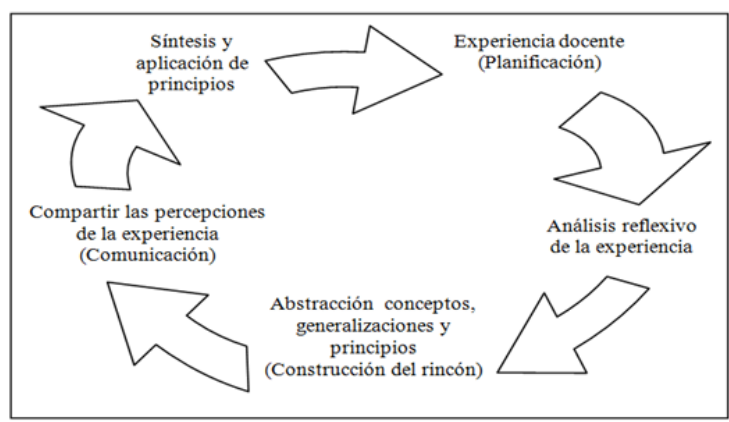

Figura 2: Adaptación de modelo de aprendizaje reflexivo experiencial de Kolb (1984).

\section{1era etapa: Planificación del rincón}

En esta etapa los estudiantes debían proponer un rincón de trabajo dirigido a niños y niñas de Educación Primaria. Debían justificar su elección y diseño en base a los conocimientos que, sobre planificación educativa y diseño curricular, habían adquirido en la asignatura. De igual modo, debían proponer aquellos recursos necesarios para el desarrollo del rincón apelando a criterios de sostenibilidad, adecuación de los materiales a las edades de los niños así como su potencial de motivación. Organizados en pequeños grupos, los estudiantes debían escoger y planificar el rincón en base a los siguientes interrogantes: 
- ¿Por qué es relevante el tema del rincón? ¿Puede interesar a alumnos de ciclo inicial de educación primaria? ¿Qué motivos respaldan la elección del tema?

- ¿Qué relación tiene el rincón con el entorno más próximo de los niños y niñas? ¿Y con su vida cotidiana? ¿Guarda alguna relación con sus inquietudes y motivaciones?

- ¿Qué queremos que aprendan los alumnos? ¿Qué tipo de aprendizaje deseamos generar? ¿Qué competencias, objetivos y contenidos podemos promover en el rincón? ¿Cómo trabajaremos? ¿Qué criterios manejamos de cara al diseño y selección de los recursos?

- ¿Cómo atenderemos a la diversidad existente en el aula?

- ¿Qué normas de uso proponemos para el rincón? ¿Por qué?

Pese a que cada grupo de estudiantes debía proponer un rincón, se realizaron sesiones conjuntas para simular la organización de una clase por rincones, de manera que todos ellos fueran diferentes, complementarios y de fácil coordinación en cuanto al tiempo que debían pasar los niños en cada uno de ellos y el tipo de actividades y habilidades que se iban a desarrollar. Esto permitió a los alumnos disponer de la experiencia del resto de compañeros, ampliando de este modo su visión de los fundamentos que rigen el trabajo por rincones.

\section{Rincón: Los personajes}

AM13O: "Escogimos esta temática porque creemos que nuestro rincón promueve el diálogo, la empatía y la resolución de conflictos. Estos tres objetivos son clave para que los alumnos puedan afrontar la realidad fuera y dentro de la escuela”

\section{Rincón: Juegos de palabras}

AM190: "Creemos que la lengua es un aspecto muy importante a trabajar en la escuela. Uno de los métodos que podemos utilizar para enseñarla pueden ser los rincones, ya que mediante los mismos podemos trabajar la comunicación, escritura, la lectura y la interpretación”

Cuadro 1. Fragmentos de las observaciones realizadas en el aula

Este proceso de análisis cooperativo condujo a los grupos a detectar algunas incoherencias en el diseño de su rincón pero también a reconocer las razones que les conducían a tomar ciertas decisiones pedagógicas en la acción. A modo de ejemplo, el cuadro 1 recoge los motivos que dieron algunos estudiantes para justificar el tema escogido en su rincón:

\section{2da etapa: Construcción del rincón}

Una vez discutida la planificación del rincón en el aula, los estudiantes procedieron a construirlo. Para ello, debían pensar y diseñar las actividades que ofrecería el rincón en base a las necesidades y realidades educativas que se pretendían abordar, las 
competencias que se deseaban fomentar, grado de dificultad de las tareas, adecuación de los recursos y temporalidad de las actividades. La construcción del rincón se realizó atendiendo a un documento de orientación elaborado por el equipo docente de la asignatura y a una rúbrica de evaluación consensuada entre profesorado y estudiantes. A modo de ejemplo, adjuntamos la rúbrica utilizada:

\begin{tabular}{|c|c|}
\hline Argumentación del rincón: & $\begin{array}{l}\text { Utiliza argumentos coherentes, críticos y precisos, con una estructura } \\
\text { y presentación claras y específicas. }\end{array}$ \\
\hline Atención a la diversidad: & $\begin{array}{l}\text { Considera diversos modos de trabajar y ritmos diferentes de } \\
\text { aprendizaje. Posibilita un trabajo heterogéneo y cooperativo. }\end{array}$ \\
\hline Organización del rincón: & $\begin{array}{l}\text { Disposición del espacio y material seleccionado son adecuados a la } \\
\text { propuesta. }\end{array}$ \\
\hline Actividades planificadas: & $\begin{array}{l}\text { Responden adecuadamente a los objetivos planteados. La secuencia y } \\
\text { nivel de dificultad adecuados. }\end{array}$ \\
\hline Objetivos del rincón: & $\begin{array}{l}\text { Están claramente definidos y son evaluables. Son relevantes y } \\
\text { responden a algún objetivo fundamental del curso. }\end{array}$ \\
\hline Coherencia del rincón: & $\begin{array}{l}\text { La relación entre los objetivos del rincón, las actividades y la } \\
\text { evaluación es coherente. }\end{array}$ \\
\hline Calidad de los aprendizajes: & $\begin{array}{l}\text { Las actividades cumplen o no con los objetivos establecidos y los } \\
\text { alumnos logran un aprendizaje significativo. }\end{array}$ \\
\hline Sistemas de control: & $\begin{array}{l}\text { Garantiza que los niños pasen por todas las actividades. Sus reglas de } \\
\text { uso y funcionamiento han sido debidamente comunicadas al } \\
\text { alumnado. }\end{array}$ \\
\hline Materiales y recursos: & $\begin{array}{l}\text { Son adecuados para la edad y nivel de los niños. Favorecen la } \\
\text { relación interpersonal y ayuda a la adquisición de nuevos } \\
\text { conocimientos. }\end{array}$ \\
\hline $\begin{array}{l}\text { Evaluación de las } \\
\text { competencias: }\end{array}$ & $\begin{array}{l}\text { En concreto, los instrumentos: } \\
\text { - Sirven para evaluar los aprendizajes. Son claros, concretos, } \\
\text { variados y conocidos por los niños. } \\
\text { - Utilizan diferentes códigos (oral, escrito, gráfico, etc.) en la } \\
\text { evaluación. } \\
\text { - Están contextualizados y adaptados a los objetivos del rincón, a la } \\
\text { metodología y a las características del grupo. } \\
\text { - Permiten una observación sistemática del trabajo de los alumnos. } \\
\text { - Evalúan todo el proceso. } \\
\text { - Valoran la construcción personal y la solidez de los aprendizajes } \\
\text { logrados. }\end{array}$ \\
\hline Presentación: & $\begin{array}{l}\text { - Uso de un lenguaje claro y fluido. } \\
\text { - Participan todos y cada uno de los integrantes del grupo. } \\
\text { - Presentan los objetivos, contenidos y materiales didácticos } \\
\text { necesarios para su desarrollo. } \\
\text { - La duración de la presentación es adecuada y fluida. }\end{array}$ \\
\hline Originalidad: & - Utilización de diversos recursos, estrategias, materiales. \\
\hline
\end{tabular}

Tabla 3. Rúbrica de evaluación de los rincones de trabajo. Curso 2012-2013. 


\section{3era etapa: Comunicación}

La presentación pública del rincón es una parte importante del proceso de simulación. Se establecieron dos sesiones de presentación, con cinco rincones cada una de ellas. De esta manera, la mitad de la clase presentaba sus rincones y el resto actuaba como alumnos, con una doble función:

- Simulando hacer las actividades de los niños, pasando por cada uno de los rincones, cumpliendo sus normas y realizando las tareas propuestas y la autoevaluación correspondiente.

- Como maestros en formación debían aplicar la rúbrica y evaluar los rincones realizados por sus compañeros.

La presentación de cada rincón se desarrolló en dos partes, una relativa al montaje real del rincón y otra consistió en una breve presentación de la propuesta de quince minutos. Se presentaron los rincones y, a continuación, los estudiantes pasaron por cada uno de ellos, participando en todas las actividades y aclarando aquellas dudas que pudieran tener respecto al planteamiento de estos.

Por último, se realizó una sesión de coevaluación, donde cada propuesta fue evaluada por toda la clase. Este proceso, de gran alcance formativo, tuvo un doble objetivo. La aplicación de los criterios de evaluación consensuados previamente entre estudiantes y profesorado y la autoevaluación del trabajo de grupo. Esta tarea implicó a todos los grupos, quienes elaboraron las retroacciones a sus compañeros enfatizando las fortalezas de su propuesta y evidenciando los aspectos a mejorar. Adjuntamos, en el siguiente cuadro, algunas reflexiones realizadas por los estudiantes en base a las retroacciones que recibieron de sus compañeros:

\section{Rincón: Somos actores y actrices}

AM11A: “...Una aspecto que se nos pasó por alto fue hacer un apartado para que los niños se autoevaluaran después de hacerlo. En la presentación, se nos señaló este error. Realmente se trataba de un grave error. Llevamos todo el curso trabajando las autoevaluaciones, además de la importancia que tienen de cara al fomento de la autocrítica y regulación de los aprendizajes”

\section{Rincón: ¡Hagamos circo!}

AM7A: "Cuando hicimos la evaluación grupal de todos los rincones, nos hicieron una observación sobre una de las actividades de nuestro rincón: los cariocas. Nos aconsejaron que esta actividad era mejor no hacerla ya que es un poco peligrosa y necesita un buen espacio para poder hacerla. Teniendo en cuenta que las aulas de primaria no son muy grandes, la actividad no era adecuada"

$$
\text { Cuadro 2. Fragmentos procedentes de los cuestionarios de autoevaluación. }
$$

Como se desprende de las reflexiones de los estudiantes, esta tercera fase es clave en el proceso reflexivo-experiencial del modelo propuesto por Kolb, en la que los estudiantes, individualmente o en grupo, son capaces de aclarar el significado de la experiencia y de tomar conciencia de cómo la información generada por la actividad se podría usar en experiencias futuras. Éste es un momento de carácter reflexivo en el 
cual la experiencia que acaba de finalizar se toma como objeto de análisis (Imbernon y Medina Moya, 2010)

\section{4rta etapa: Síntesis}

Superadas las fases anteriores, cada grupo debía llevar a cabo una identificación de lo que puede ser extrapolable a situaciones futuras. Como reflexión sobre la práctica realizada, cada grupo de estudiantes elaboró un informe escrito, que contemplaba la valoración de la tarea realizada y la síntesis de los aprendizajes obtenidos. Para ello, se basaron en la reflexión conjunta del trabajo realizado (autoevaluación) y en la evaluación y opiniones de sus compañeros en la sesión de evaluación compartida (coevaluación).

\section{Conclusiones}

Los resultados obtenidos, derivados de nuestra participación como docentes en la experiencia de los rincones, nos hacen concluir que el trabajo experiencial en la formación de maestros debe ir necesariamente unido a la reflexión. Sin ella, el sentido y la intencionalidad de la acción -que conlleva el trabajo experiencial- se vuelve vago y confuso. La reflexión, que implica la inmersión consciente del estudiante en el mundo de su experiencia (Pérez Gómez, 1989), le permite adaptarse y transformar nuevas situaciones problemáticas al reconocer sus necesidades y dificultades. Cabe señalar que la propuesta de los rincones de trabajo se presenta como una estrategia viable y adecuada para esta finalidad. Conduce al estudiante a un trabajo experiencial basado en la acción y la reflexión y, en el caso particular de nuestra innovación, ha potenciado también el aprendizaje cooperativo.

Basándonos en nuestra experiencia, podemos señalar que la puesta en marcha de los rincones de trabajo exige una planificación flexible ante las contingencias que puedan ir apareciendo a lo largo de la experiencia (de carácter único, impredecibles e irrepetibles) Es también necesario proporcionar ambientes seguros y potenciadores de sentido y significado, donde los estudiantes puedan experimentar sin miedo, desarrollar sus ideas y reflexionar sobre ellas con el acompañamiento del docente. Como es natural, en la experiencia de los "rincones de trabajo" la organización y la configuración del aula se convierten en aspectos cruciales. En este sentido, debe ser pensada y configurada de tal manera que ofrezca condiciones de aprendizaje que sitúen al alumnado en un contexto práctico que simule el oficio de maestro.

Es oportuno también señalar que para el buen funcionamiento de los rincones de trabajo son necesarios los siguientes aspectos: un tiempo de aprendizaje dilatado y superior al que puede ser necesario en otro tipo de estrategias, recursos ajustados a cada propuesta de rincón, espacios educativos amplios y mobiliario móvil así como también medios de comunicación, constantes y eficientes, entre los diversos agentes implicados (profesor-alumnos y alumnos-alumnos) 
En concreto,los rincones de trabajo conducen a la configuración de un aula compleja que demanda el uso de diferentes estrategias en función de los objetivos de cada etapa (Etapas: $1^{\mathrm{a}}$ Panificación del rincón, $2^{\mathrm{a}}$ Construcción del rincón, $3^{\mathrm{a}}$ Comunicación del rincón y $4^{\mathrm{a}}$ Síntesis). Según la opinión de los estudiantes, las estrategias implementadas a lo largo de la experiencia poseen un elevado alcance pedagógico. El inicio del trabajo con preguntas que dirijan a los estudiantes a cuestionarse sobre los motivos que justifican su propuesta; la construcción dialogada y consensuada de una rúbrica de evaluación; el montaje y la puesta en práctica del rincón en el aula; el trabajo de reflexión y síntesis sobre la actividad realizada; el trabajo en equipo y el seguimiento y tutorización constante durante todo el proceso han sido aspectos que han recibido una valoración altamente positiva por parte de los estudiantes.

Finalmente, concluimos que la experiencia de los rincones ofrece condiciones didácticas óptimas para la articulación de conocimientos teóricos y prácticos, la construcción de conocimientos profesionales y el inicio de una práctica docente reflexiva. Los rincones de trabajo no potencian únicamente la adquisición de contenidos sino que se dirigen a ayudar al estudiante a pensar y recuperar, de manera articulada, todos sus saberes en torno a una situación práctica, potenciando además el vínculo entre teoría y práctica. Una vez finalizada la experiencia, consideramos que los rincones de trabajo potencian procesos cognitivos de orden superior como la reflexión, la crítica, el análisis y la síntesis así como la resolución de problemas, conducen a importantes mejoras en el ámbito de la comunicación oral y escrita y promueven la motivación, implicación y la autonomía del alumnado en su propio proceso de aprendizaje.

\section{Referencias bibliográficas}

ÁLVAREZ, C. (2013). ¿Puede superar el profesorado la rutpura teoría-práctica. Un estudio de caso. REICE, 11(4) Disponible en: http://www.rinace.net/reice/numeros/arts/vol11num4/art7_htm.htm

ANTÚNEZ MARCOS, S. (1999). El trabajo en equipo de los profesores y profesoras: factor de calidad, necesidad y problema. El papel de los directivos escolares. Educar, 24, 89-110. Disponible http://diposit.ub.edu/dspace/bitstream/2445/22305/1/500889.pdf.

BARBA MARTÍN, J.J., MARTÍNEZ SCOTT, S. Y TORREGO EGIDO, L. (2012). El Proyecto de aprendizaje tutorado cooperativo. Una experiencia en el grado de maestra de Educación Infantil. Revista de Docencia Universitaria. REDU. Monográfico: Buenas prácticas docentes en la enseñanza universitaria, 10 (1), 123-144.

COCHRAN-SMITH, M. Y LYTLE, S. (2003). Más allá de la certidumbre: adoptar una actitud indagadora sobre la práctica. En Lieberman, A. y Miller, L. (Cood). La indagación como base de la formación del profesorado y la mejora de la educación (pp. 65-79). Barcelona: Octaedro 
DEWEY, J. (1916). Democracy and Education. Londres: Macmillan (Trad. Cast: Democracia y educación. Madrid, Morata, 2001).

ELLIOTT, J. (1990). La investigación-acción en educación. Madrid: Morata.

FLORES MARTÍNEZ, P. (1998). Formación inicial de profesores de matemáticas como profesionales reflexivos. UNO. Revista de Didáctica de las Matemáticas, núm. 17, 37-48. Disponible en: http://www.ugr.es/ pflores/textos/aRTICULOS/Investigacion/UNO98.pdf.

GRIFFIN, M. (2003). Using critical incidents to promote and assess reflective thinking in preservice teachers. Reflective Practice: International and Multidisciplinary Perspectives, 4(2), 207-220. Doi: 10.1080/14623940308274

HERRERA CAPITA, A.M. (2009). Los rincones en Educación Primaria. Revista Digital Innovación y experiencias educativas, 15, 1-8. Disponible en: http://www.csicsif.es/andalucia/modules/mod_ense/revista/pdf/Numero_15/ANGELA\%20MARI A_HERRERA_2.pdf.

IMBERNON MUÑOZ, F. Y MEDINA MOYA, J.L. (2008). Metodología participativa en el aula universitaria. La participación del alumnado. Barcelona: Octaedro.

JOHNSON, D.; JOHNSON, J. Y HOLUBEC, E. (1999). El aprendizaje cooperativo en el aula. Buenos Aires: Paidós.

KOLB, D. (1984). Experiential Learning. Londres: Prentice-Hall.

KORTHAGEN, F. (2010). La práctica, la teoría y la persona en la formación del profesorado. Revista Interuniversitaria de Formación del Profesorado, 68(24,2), 83-101.Disponible en: http://www.aufop.com/aufop/uploaded_files/articulos/1279236671.pdf.

LATORRE BELTRÁN, A.; DEL RINCÓN IGEA, D. Y ARNAL AGUSTÍN, J. (1996). Bases metodológicas de la investigación educativa. Barcelona: GR92.

PÉREZ GÓMEZ, A. (2010). Aprender a educar. Nuevos desafíos para la formación de docentes. Revista Interuniversitaria de Formación del Profesorado, 68(24,2), 3760. Disponible en: http://www.redalyc.org/articulo.oa?id=27419198003.

PÉREZ GÓMEZ, A. (1989). Paradigmas contemporáneos en investigación didáctica. En Gimeno, Sacristán, J. y Pérez Gómez, A.: La enseñanza: su teoría y su práctica (pp. 95-139). Madrid: Akal.

PERRENOUD, P. (2001). La formación de los docentes en el siglo XXI. Revista de Tecnología Educativa, 24(3), 503-533. Disponible en: http://www.unige.ch/fapse/SSE/teachers/perrenoud/php_main/php_2001/2001_36.h tml.

PIQUÉ SIMÓM, B.; COMAS ARBÒS, A. Y LORENZO RAMÍREZ, N. (2010). Estratègies de pràctica reflexiva en la formació inicial de mestres d'educació infantil. Barcelona: Graó. 
RODRÍGUEZ TORRES, J. (2011). Los rincones de trabajo en el desarrollo de competencias básicas. Revista Docencia e Investigación, 21, 105-130. Disponible en:

http://www.uclm.es/varios/revistas/docenciaeinvestigacion/pdf/numero11/06.pdf.

RUSSELL, T. (2005). Can reflective practice be taught? Reflective Practice: International and Multidisciplinary Perspectives, 6(2), 199-204.

SANDÍN, M.P. (2003). Investigación cualitativa en educación. Madrid: McGrawHill.

SALEH, T. (2011). Statistical Analysis of Cooperative Strategy Compared with Individualistic Strategy: An Application Study. The Journal of Effective Teaching, 11(1), 19-27

SCHÖN, D. (1983). The reflective practitioners: How Professionals Think in Action. New York: Basic Books.

SCHÖN, D. (1992). La formación de profesionales reflexivos: Hacia un nuevo diseño de la enseñanza y el aprendizaje en las profesiones. Barcelona: Paidós.

TRAN, V. y LEWIS, R. (2012). Effects of cooperative learning on students at an Giang University in Vietnam. International Education Studies, 5(1), 86-99.

ZEICHNER, K. (2010). Nuevas espistemologías en formación del profesorado. Repensando las conexiones entre las asignaturas del campus y las experiencias de prácticas en la formación del profesorado en la universidad. En Pérez Gómez, A. (ccord.): Reinventar la profesión docente (pp. 123-149). Zaragoza: AUFOP. 


\section{Correspondencia con los autores}

$\mathrm{M}^{\mathrm{a}}$ Teresa COLÉN RIAU

Universidad de Barcelona

Departamento de Didáctica y Organización Educativa

Campus Mundet

Edifici Llevant, 2a planta

Passeig de la Vall Hebrón, 171

08035 Barcelona

e-mail: mcolen@ub.edu

Beatriz JARAUTA BORRASCA

Departament de Didàctica i Organizació Educativa

Campus Mundet

Edifici Llevant, 2a planta

Passeig de la Vall d'Hebron, 171

08035 Barcelona

e-mail: bjarauta@ub.edu

Leyla Claudina CASTRO GONZÁLEZ

Departament de Didàctica i Organizació Educativa

Campus Mundet

Edifici Llevant, 2a planta

Passeig de la Vall d'Hebron, 171

08035 Barcelona

e-mail: leyla_castro@ub.edu 